\title{
因HAD
}

DOI: http://doi.org/10.22585/hospdomic.v5i2.129

\section{Calidad de vida de los cuidadores de las personas adultas en ingreso domiciliario: revisión sistemática}

\section{Quality of life of caregivers of adult people at home admission: systematic review}

Salima Alouat-Chentouf' (1) 0000-0002-1829-9472

Javier Sanz-Valero ${ }^{2,3}$ (1) 0000-0002-8735-0075

1. Universidad Miguel Hernández. Facultad de Farmacia, Campus de Sant Joan d'Alacant. Alicante. España.

2. Instituto de Salud Carlos III. Escuela Nacional de Medicina del Trabajo. Madrid. España.

3. Universidad Miguel Hernández. Departamento de Salud Pública e Historia de la Ciencia, Campus de Sant Joan d'Alacant. Alicante. España.

Correspondencia/Correspondence

Salima Alouat-Chentouf

salima.aloua!@gmail.com

Recibido/Received

14.01 .2021

Aceptado/Accepted

11.02 .2021

Conflicto de Intereses/Competing interest

La autora y el autor de la presente revisión declaran la

inexistencia de conflicto de interés.
Financiación/Funding

Sin financiación

Contribuciones de autoría/Author contributions La autora y el autor de la presente revisión contribuyeron por igual en la concepción y el diseño, SAC se encargó de la adquisición de datos, ambos realizaron el análisis e interpretación, y la redacción del primer borrador de la revisión, JSV realizó la revisión crítica del contenido y los dos aprobaron la versión final que fue remitida para su publicación.

CÓMO CITAR ESTE TRABAJO | HOW TO CITE THIS PAPER

Alouat-Chentouf S, Sanz-Valero J. Calidad de vida de los cuidadores de las personas adultas en ingreso domiciliario: revisión sistemática. Hosp Domic. 2021;5(2):89-1 13. 


\section{RESUMEN}

Objetivo: Revisar la documentación científica relacionada con la calidad de vida (CV) de los cuidadores de las personas adultas en ingreso domiciliario.

Método: Revisión crítica y sistemática. Los datos se obtuvieron de la consulta a las bases de datos bibliográficas: MEDLINE (vía PubMed), Cochrane Library y Embase. Los términos utilizados, como descriptores y como texto en los campos de registro del título y el resumen, fueron «Quality of Life», "Caregivers» y «Home Care Services», utilizando los filtros «Humans», «Adult: 19+ years» y «Clinical Trial». Fecha de la última actualización de la búsqueda octubre de 2020. La calidad documental de los artículos se evaluó mediante el cuestionario CONSORT.

Resultados: De las 217 referencias recuperadas, tras aplicar los criterios de inclusión y exclusión, se seleccionaron 29 ensayos clínicos. Las puntuaciones CONSORT oscilaron entre mínimo de 9,5 y máximo de 20,5 con mediana de 16 (puntuación máxima posible de 21). Según los criterios SIGN esta revisión presentó evidencia «1+» (revisiones sistemáticas de ensayos clínicos aleatorizados con bajo riesgo de sesgos) con un grado de recomendación A. Los cuestionarios más utilizados para valorar la CV fueron los genéricos European Quality of Life5 Dimensions (EQ-5D) y el Short Form Health Survey 36 items (SF-36), en 4 ocasiones. La intervención que se realizó con una mayor frecuencia fue la formación de los cuidadores, en 10 ensayos.

Conclusiones: Formar y ayudar al cuidador/a supuso una mejoría en su la calidad de vida y en la mejoría de los niveles de ansiedad. Aunque, en algunos ensayos no se encontraron diferencias entre el grupo de la intervención y el grupo control. El hecho de intervenir (preocuparse del cuidador/a) motivó una menor carga en el estado anímico del mismo, aunque sin resultados claramente significativos.

Palabras clave: Calidad de Vida; Cuidadores; Servicios de Atención de Salud a Domicilio; Encuestas y Cuestionarios; Educación Continua.

\section{ABSTRACT}

Objective: To review the scientific documentation related to the quality of life (QoL) of caregivers of adults in home care services.

Method: Critical and systematic review. The data were obtained from consulting the bibliographic databases: MEDLINE (via PubMed), Cochrane Library and Embase. The terms used, as descriptors and as text in the title and abstract registration fields, were "Quality of Life», «Caregivers» and «Home Care Services», using the filters «Humans», "Adult: 19+ years "And" Clinical Trial ". Date of the last update of the search October 2020. The documentary quality of the articles was evaluated using the CONSORT questionnaire.

Results: From 217 references retrieved, after applying the inclusion and exclusion criteria, 29 clinical trials were selected. CONSORT scores ranged from a minimum of 9.5 to a maximum of 20.5 with a median of 16 (maximum possible score of 21). According to the SIGN criteria, this review presented evidence «1+» (systematic reviews of randomized clinical trials with low risk of bias) with a grade of recommendation A. The most used questionnaires to assess QoL were the generic European Quality of Life-5 Dimensions (EQ-5D) and the Short Form Health Survey 36 items (SF-36), on 4 occasions. The intervention that was carried out with the highest frequency was the training of caregivers, in 10 trials.

Conclusions: Training and helping the caregiver led to an improvement in their quality of life and an improvement in anxiety levels. Although, in some trials no differences were found between the intervention group and the control group. The fact of intervening (worrying about the caregiver) led to a lesser burden on their mood, although without clearly significant results.

Keywords: Quality of Life; Caregivers; Home Care Services; Surveys and Questionnaires; Education, Continuing. 


\section{INTRODUCCIÓN}

El concepto de calidad de vida relacionado con la salud (CVRS) se contextualizó en relación a como la enfermedad (como fuente de dolor, disfunción física e incomodidad) imponía limitaciones o alteraciones en el comportamiento diario, actividades sociales y bienestar psicológico, así como en otros aspectos de la vida cotidiana ${ }^{(1)}$.

Según la Organización Mundial de la Salud, el concepto de salud se conoce como un estado completo de bienestar físico, mental y social, y no solamente la ausencia de enfermedad, pero nos encontramos con un constructo multidimensional, no observable directamente, ya que va a depender de la percepción de cada individuo y que solo puede ser deducido a través de indicadores generados a partir de cuestionarios ${ }^{(2-4)}$.

La medición de la calidad de vida aporta una dimensión holística al curso del estado clínico o a la respuesta a una intervención determinada. Además, medir la CVRS es un proceso complejo que responde a las expectativas individuales en diferentes facetas de la vida. La forma en que la CVRS se ve afectada es un tema de constante interés y debate permanente ${ }^{(5)}$.

Gracias a los cambios sociodemográficos y los avances científicos, a lo largo del tiempo, se ha experimentado un cambio en la longevidad y el transcurso de muchas enfermedades que antes eran rápidamente mortales, convirtiéndose ahora en enfermedades crónicas, o en su defecto, en enfermedades con las que se ha conseguido alargar el periodo de vida junto con un deterioro más lento de la calidad de vida relacionado con la salud((6).

Esta situación, motivó que muchos países establecieran políticas sanitarias basadas en trasladar los recursos al hogar de los enfermos, con la finalidad de que se reduzca el coste de la factura sanitaria y, a la vez, mejorar la calidad vida y la atención de los enfermos en comparación con aquellos que se encuentran bajo el cuidado de los centros sanitarios ${ }^{(7)}$.

Además, la hospitalización a domicilio $(\mathrm{HaD})$ aporta ventajas como otra modalidad de hospitalización distinta a la convencional. Existe una contrastada evidencia acerca de dichas ventajas en lo que respecta a la eficacia, seguridad y al alto nivel de satisfacción de esta modalidad asistencial. Los factores clave para su correcta implementación son garantizar una adecuada selección de los pacientes dependientes de $\mathrm{HaD}$ y disponer de un equipo experimentado en atención de casos complejos en el domicilio(8).

Así, la $\mathrm{HaD}$ se define como un servicio que proporciona tratamiento activo por profesionales de la salud en el hogar del paciente, por una afección que de otro modo requeriría atención hospitalaria. La implementación de los servicios hospitalarios en el hogar tiene también como objetivo mejorar la percepción del paciente respecto al tratamiento y mejorar su calidad de vida al ofrecer la posibilidad de dicha atención en un ambiente amigable y con la misma eficiencia en la atención médica ${ }^{(9)}$. Ahora bien, en este tipo asistencial, la figura del cuidador adquiere una importancia capital.

El cuidador es un elemento crítico de la hospitalización domiciliaria, habiéndose encontrado gran correlación entre el nivel de salud física y psicológica del cuidador con la calidad de la atención domiciliaria, reconociéndose así necesidades tanto en el cuidador como en el enfermo(10).

El cuidado no profesional sigue siendo mayoritario y tiene una gran relevancia social, pues sirve de apoyo a las personas en situación de dependencia y constituye uno de los recursos más importantes para la política social y sanitaria al reducir la utilización de recursos asistenciales. Sin embargo, asumir el cuidado de un ser querido puede llegar a afectar el propio bienestar psicológico(11). Ahora bien, estos cuidados son de suma importancia ya que, al estudiar la relación, con respecto a 
la evaluación del estado de salud, se observó una adecuada concordancia de lo percibido entre los pacientes y su principal cuidador, sobre todo si era mujer y familiar en primer grado(6).

Asimismo, la presencia del cuidador brinda apoyo emocional, creando sentimientos de tranquilidad y seguridad en el paciente y es un vínculo de comunicación entre los familiares y los profesionales sanitarios. Por lo tanto, el cuidador es una figura clave en el proceso de salud-enfermedad, ya que este asume una parte del cuidado del paciente tornándose fundamental para la salud de las personas y como un apoyo para enfrentarse al proceso de la hospitalización domiciliaria ${ }^{(12)}$.

El cuidador es una persona fundamental en las alternativas del cuidado domiciliario, pero también debe ser receptor de atención. En consecuencia, el objetivo de este estudio fue revisar y analizar críticamente la documentación científica relacionada con la calidad de vida de los cuidadores de las personas adultas en ingreso domiciliario.

\section{MÉTODOS}

\section{Diseño}

Estudio descriptivo transversal y análisis crítico de los trabajos recuperados mediante revisión sistemática.

\section{Fuente de obtención de los datos}

Los datos se obtuvieron de la consulta directa y acceso, vía Internet, a las siguientes bases de datos bibliográficas del ámbito de las ciencias de la salud: MEDLINE (vía PubMed), Embase y Cochrane Library.

La selección de estas tres bases de datos se debió a que todas ellas cuentan con un lenguaje normalizado de indización (Thesaurus), lo que dota a la búsqueda bibliográfica de una mayor pertinencia.

\section{Unidad de análisis}

Se trabajó con los artículos publicados y recuperados desde la base de datos bibliográficas indicadas.

Para evitar posibles duplicidades no se tuvieron en cuenta las comunicaciones a congresos, aunque sean de gran importancia para la comunidad investigadora ya que contribuyen a la rápida comunicación y difusión de la investigación.

\section{Tratamiento de la información}

Para definir los términos de la búsqueda se consultó el Thesaurus desarrollado por la U.S. National Library of Medicine. Se consideró adecuado el uso de los términos «Quality of Life», "Caregivers» $y$ «Home Care Services», como descriptores y como texto en los campos de registro del título y el resumen, conformando la sintaxis de búsqueda final mediante la intersección booleana de tres ecuaciones: (Ecuación 1: Población) AND (Ecuación 2: Intervención) AND (Ecuación 3: Resultado). 


\section{Población: Cuidadores (Caregivers)}

"Caregivers"[Mesh] OR "Caregiver"[Title/Abstract] OR "Carer"[Title/Abstract] OR "Care Giver"[Title/Abstract] OR "Spouse Caregiver"[Title/Abstract] OR "Family Caregiver"[Title/Abstract] OR "Keeper"[Title/Abstract]

\section{Intervención: Servicios de Atención de Salud a Domicilio (Home Care Services)}

"Home Care Services"[MeSH] OR "Home Care"[Title/Abstract] OR "Domiciliary Care"[Title/ Abstract] OR "Home Care Services, Hospital- Based"[Mesh] OR "Hospital-Based Home Care"[All Fields] OR "Hospital Based Home Care"[Title/Abstract] OR "Hospital Home Care Services"[Title/ Abstract] OR "Hospital-Based Home Care Services"[Title/Abstract] OR "Hospital Based Home Care Services"[Title/Abstract] OR "Home Hospitalization"[Title/Abstract] OR "Hospital at Home"[Title/Abstract] OR "Hospital-at-Home"[Title/Abstract] OR "Hospital Home Care"[Title/Abstract] OR "Hospital at Home Care"[Title/Abstract] OR "Hospital in the Home"[Title/Abstract]

\section{Resultado: Calidad de vida (Quality of Life)}

"Quality of Life"[Mesh] OR "Quality of Life"[Title/Abstract] OR "Life Quality"[Title/Abstract] OR "Health Related Quality Of Life"[Title/Abstract] OR "HRQOL"[Title/Abstract]

La ecuación de búsqueda final se desarrolló para su empleo en la base de datos MEDLINE, vía PubMed, utilizando los filtros: "Humans», "Adult: 19+ years» y "Clinical Trial».

Esta estrategia se adaptó a las características de las otras dos bases de datos consultadas. La búsqueda se realizó desde la primera fecha disponible, de acuerdo a las características de cada base de datos, hasta octubre de 2020. Adicionalmente, como búsqueda secundaria y para reducir los posibles sesgos de publicación, se examinó el listado bibliográfico de los artículos que fueron seleccionados en la búsqueda principal, con el objeto de identificar estudios no detectados en la revisión (búsqueda manual).

\section{Selección final de los artículos}

Se escogieron para la revisión y análisis crítico los artículos que cumplieron los siguientes criterios de inclusión: adecuarse a los objetivos de la búsqueda (intervención psicológica), ser ensayo clínico y estar publicados en revistas arbitradas por pares y escritos en inglés, español o portugués.

Se excluyeron: aquellos artículos que, además, no se pudo encontrar el texto completo del mismo, no existió relación entre la intervención y el resultado a estudio y los que incluían población no adulta (menos de 18 años).

La selección de los artículos pertinentes se realizó de forma independiente por los autores: SACH y JSV. Para dar por válida la inclusión de los estudios se estableció que la valoración de la concordancia entre estos autores (índice Kappa) debía ser superior al 60\% (fuerza de la concordancia buena o muy buena). Siempre que se cumpliera esta condición, las posibles discordancias se solucionaron mediante el consenso entre ambos autores. 


\section{Calidad de los documentos seleccionados}

Para valorar la calidad de la presentación de los documentos seleccionados se utilizaron las directrices para la comunicación de los ensayos clínicos CONSORT (CONsolidated Standards of Reporting Trials) ${ }^{(13)}$, que contiene un listado de 25 aspectos esenciales que deben describirse en la publicación de estos estudios. Para cada artículo seleccionado se asignó un punto por cada ítem presente (en caso de no ser aplicable no puntuaba, NA = No Aplica). Cuando un ítem estaba compuesto por varios puntos, estos se evaluaron de forma independiente, dándole el mismo valor a cada uno de ellos y posteriormente se realizó un promedio (siendo éste el resultado final de ese ítem), de tal forma que en ningún caso se pudiera superar la puntuación de un punto por ítem. No se tuvo en cuenta (no aplicaban) los ítems 9 (mecanismo de ocultación de la asignación, 10 (implementación) y 11 (enmascaramiento) del cuestionario, al no considerarse necesarias las etapas de ocultación y de cegamiento en el tipo de intervención estudiado. Tampoco se consideraron necesarios, para esta revisión, los resultados del análisis de subgrupos, ítem 18. En consecuencia, el valor final y su porcentaje se calcularon sobre 21 ítems.

Para conocer el nivel de evidencia y su grado de recomendación se utilizaron las recomendaciones de Scottish Intercollegiate Guidelines Network (SIGN) ${ }^{(14)}$.

\section{Extracción de los datos}

El control de la corrección de los datos se realizó mediante dobles tablas que permitieron la detección de las desviaciones y su subsanación mediante nueva consulta de los originales.

Para determinar la actualidad de los artículos se calculó el semiperíodo de Burton-Kebler (la mediana de la edad) y el Índice de Price (porcentaje de artículo con edad inferior a los 5 años).

Los estudios se agruparon según las variables a estudio, con el fin de sistematizar y facilitar la comprensión de los resultados, considerando los siguientes datos: primer autor y año de publicación, diseño del estudio (tipo de ensayo clínico), país donde se efectuó el estudio, Idioma de publicación del documento, población incluida en los artículos, periodo de implementación de la intervención, intervención realizada y resultado principal obtenido en los ensayos revisados.

\section{RESULTADOS}

Al aplicar los criterios de búsqueda se recuperaron un total de 217 referencias: 88 (40,6\%) en MEDLINE (vía PubMed), 75 (34,6\%) en Embase y 54 (24,9\%) en Cochrane Library. Tras depurar los 76 $(35,0 \%)$ registros repetidos y aplicar los criterios de inclusión y exclusión, fue posible seleccionar 29 $(13,4 \%)$ artículos ${ }^{(15-43)}$, para su revisión y análisis crítico; ver figura 1 y tabla 1. 
Figura 1 - Flujograma de la identificación y selección de los estudios

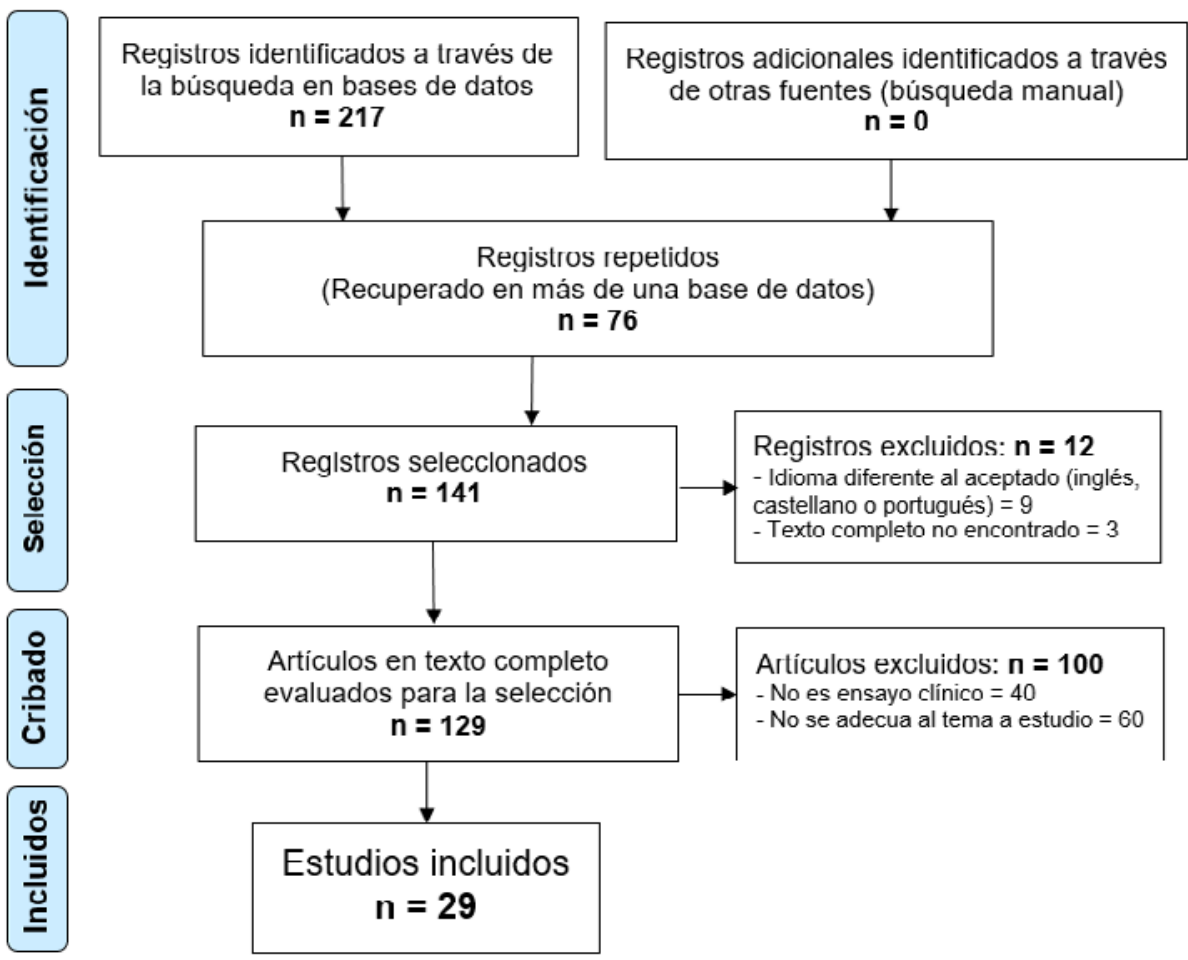

El acuerdo sobre la pertinencia de los estudios seleccionados, entre los dos evaluadores, calculado mediante el índice Kappa, fue del 100\%.

Los ensayos elegidos presentaron una obsolescencia, según el Índice de Burton-Kebler, igual a 12,0 años, con un Índice de Price del 24,1\% que se corresponde con los 7 ensayos, publicados a partir del año 2016 $6^{(15-21)}$. El año con mayor número de trabajos publicados fue el 2004, con 4 publicaciones $^{(36-39)}$, el $12,5 \%$ del total de estudios incluidos en la revisión. 
Tabla 1. Resumen de los estudios revisados sobre calidad de vida de los cuidadores de las personas adultas en ingreso domiciliario

\begin{tabular}{|c|c|c|c|c|c|c|c|}
\hline Primer autor y año & País & Idioma & Población & Periodo & $\begin{array}{l}\text { Método de evaluar la calidad } \\
\text { de vida }\end{array}$ & Intervención & Resultado principal \\
\hline $\begin{array}{l}\text { Demiris et al., } \\
2019^{(15)}\end{array}$ & EE. UU. & Inglés & $\begin{array}{l}\text { N total }=514 \\
\text { H/M =128/386 } \\
\text { edad media = } 60,3 \text { años } \\
\text { hijos adultos: } 55 \%, \\
\text { cónyuge/parea: } 27 \% \text {, otros: } 18 \% ; \\
\text { Gac (llamada amistosa) = } 172 \\
\text { H/M = 40/132 } \\
\text { Edad media: } 59,7 \\
\text { hijos adultos: } 88, \\
\text { cónyuge o pareja: } 50 \text {, otros: } 34 ; \\
\text { Gf2f (cara a cara) = } 171 \\
\text { H/M = 46/125 } \\
\text { Edad media: } 59,9 \\
\text { hijos adultos: } 100, \\
\text { cónyuge o pareja: } 39 \text {, otros: } 32 ; \\
\text { Grc (videoconferencia) = 171 } \\
\text { H/M = 42/129 } \\
\text { Edad media: } 61,3 \\
\text { hijos adultos: } 96, \\
\text { cónyuge o pareja: } 50, \text { otros: } 25 ;\end{array}$ & 4 años & $\begin{array}{l}\text { Índice de calidad de vida del } \\
\text { cuidador revisado (CQLI-R); } \\
\text { incluye } 4 \text { dimensiones: fisica, } \\
\text { social, emocional y financiera. }\end{array}$ & $\begin{array}{l}\text { Estas intervenciones se } \\
\text { han realizado mediante } 3 \\
\text { formas diferentes: llamada } \\
\text { amistosa, cara a cara y } \\
\text { videoconferencia. }\end{array}$ & $\begin{array}{l}\text { Reducción en cada una de las } \\
\text { subescalas del CQLI-R de } \\
\text { cuidadores en Gf2f. }\end{array}$ \\
\hline von Känel et al., 2019(16) & EE. UU. & Inglés & $\begin{array}{l}\mathrm{N} \text { total }=134 \text { (cónyuges) } \\
\mathrm{H} / \mathrm{M}=29 / 105 \\
\text { Edad media = } 74,1 \text { años }\end{array}$ & No consta & Entrevista de una sola pregunta & $\begin{array}{l}\text { Comparación } \\
\text { cuidadores con salud } \\
\text { autoevaluada deficiente / } \\
\text { regular con aquellos con } \\
\text { salud autoevaluada buena, } \\
\text { muy buena o excelente }\end{array}$ & $\begin{array}{l}\text { Cuidadores con autoevaluación } \\
\text { deficiente / regular se } \\
\text { caracterizaron por niveles más } \\
\text { altos de comorbilidad médica, baja } \\
\text { función física, alto afecto negativo y } \\
\text { mayores factores estresantes. }\end{array}$ \\
\hline Gitlin et al., 2018 (17) & EE. UU. & Inglés & $\begin{array}{l}N \text { total }=160 \\
H / M=4 / 156 \\
\text { Edad media }=72,4 \\
\text { Cónyuges/no cónyuges = 139/21 } \\
\text { Gi }=76 \\
G c=84\end{array}$ & 4-8 meses & $\begin{array}{l}\text {-Escala de Depresión (CESD-10) } \\
\text {-Formulario corto de carga de } \\
\text { Zarit } 12 \text { ítems }\end{array}$ & $\begin{array}{l}\text { Programa de actividades } \\
\text { en el hogar mediante } 8 \\
\text { sesiones telefónicas }\end{array}$ & $\begin{array}{l}\text { Alivio angustia del cuidador en Gi } \\
\text { en los } 4 \text { meses. Beneficios no se } \\
\text { extendieron a los } 8 \text { meses. }\end{array}$ \\
\hline Bilgin et al., 2018(18) & Turquía & Inglés & $\begin{array}{l}\mathrm{N} \text { total }=72 \\
\mathrm{Gi}=34 \\
\mathrm{H} / \mathrm{M}=15 / 19 \\
\text { Edad media }=41,70 \\
\text { Padre }=1 \\
\text { Esposa }=15 \\
\text { Hermano-hijo/otros }=18 \\
\mathrm{GC}=38 \\
\mathrm{H} / \mathrm{M}=12 / 26 \\
\text { Edad media }=40,10 \\
\text { Padre }=1 \\
\text { Esposa }=12 \\
\text { Hermano-hijo/otros }=25\end{array}$ & $\begin{array}{l}3^{\circ} \text { día tras el } 5^{a} \\
\text { día del tratamiento } \\
\text { quimioterápico (en } \\
\text { visitas cada } 28 \text { días), sin } \\
\text { mencionar el número de } \\
\text { visitas }\end{array}$ & $\begin{array}{l}\text { Escala de calidad } \\
\text { de vida del cuidador: cáncer } \\
\text { (CQOLC) }\end{array}$ & Cuatro visitas domiciliarias & $\begin{array}{l}\text { La calidad de vida en general del } \\
\text { Gi mejoró. }\end{array}$ \\
\hline
\end{tabular}




\begin{tabular}{|c|c|c|c|c|c|c|c|}
\hline Primer autor y año & País & Idioma & Población & Periodo & $\begin{array}{l}\text { Método de evaluar la calidad } \\
\text { de vida }\end{array}$ & Intervención & Resultado principal \\
\hline Brijoux et al., 2016 & Alemania & Inglés & $\begin{array}{l}\mathrm{N} \text { total }=73 \\
\mathrm{H} / \mathrm{M}=19 / 54 \\
\text { Edad media }=64,26 \\
\text { Gapoyo al familiar }=39 \\
\mathrm{H} / \mathrm{M}=11 / 28 \\
\text { Edad media }=62,41 \\
\text { Cónyyge }=21 \\
\text { Padre-suegro }=16 \\
\text { Hermano-hermana }=1 \\
\text { Otro }=1 \\
\text { Gapoyo al cuidador convencional = } 34 \\
\mathrm{H} / \mathrm{M}=8 / 26 \\
\text { Edad media }=66,12 \\
\text { Cónyuge }=22 \\
\text { Padre-suegro }=11 \\
\text { Hermano-hermana }=1 \\
\text { Otro = }\end{array}$ & 2 años y 1 mes & $\begin{array}{l}\text {-Escala salud mental y física } \\
\text { (SF-12) } \\
\text {-Inventario de Berlín para } \\
\text { la carga de los cuidadores } \\
\text { con pacientes con demencia } \\
\text { (BIZA-D) }\end{array}$ & $\begin{array}{l}\text { Apoyo y formación a ambos } \\
\text { grupos. }\end{array}$ & $\begin{array}{l}\text { El Gapoyo al familiar mejoró su } \\
\text { calidad de vida relacionada con } \\
\text { la salud en comparación con el } \\
\text { Gapoyo al cuidador convencional, } \\
\text { también se experimentó una } \\
\text { reducción de carga de la atención } \\
\text { informal. }\end{array}$ \\
\hline $\begin{array}{l}\text { Kuo et al., } \\
2016^{(20)}\end{array}$ & China & Inglés & $\begin{array}{l}\text { Gi Alzheimer }=30 \\
\text { H/M }=8 / 22 \\
\text { Edad media }=54,60 \\
\text { Cónnyge }=6 \\
\text { Hijo/a }=24 \\
\text { Gc }=38 \\
H / M=11 / 27 \\
\text { Edad media = } \\
\text { Cónyuge }=12 \\
\text { Hijo/a }=26 \\
\text { Gi Demencia Vascular = } 25 \\
\text { H/M }=5 / 20 \\
\text { Edad media = } 54.64 \\
\text { Cónyuge }=9 \\
\text { Hijo/a }=16 \\
\text { Gc }=21 \\
H / M=5 / 16 \\
\text { Edad media }=55.86 \\
\text { Cónyuge }=4 \\
\text { Hijo/a }=17\end{array}$ & 18 meses & $\begin{array}{l}\text {-CESD-20 } \\
\text {-SF-36 }\end{array}$ & Programa de formación & $\begin{array}{l}\text { Intervención mayoritariamente } \\
\text { beneficiosa en cuidadores de } \\
\text { pacientes con Alzheimer, mejor } \\
\text { calidad de vida y disminución de } \\
\text { sintomas depresivos. }\end{array}$ \\
\hline $\begin{array}{l}\text { Prick et al., } \\
2016^{(21)}\end{array}$ & EE. UU. & Inglés & $\begin{array}{l}N \text { total }=111 \\
H / M=31 / 80 \\
\text { Edad media }=72 \\
\text { Cónyuge }=100 \\
\text { Hijo/otro }=11 \\
\text { Gi }=57 \\
H / M=19 / 38 \\
\text { Edad media }=73 \\
\text { Cónyuge }=50 \\
\text { Hijo/otro }=7 \\
\mathrm{Gc}=54 \\
\mathrm{H} / \mathrm{M}=12 / 42 \\
\text { Edad media }=71 \\
\text { Cónyuge }=50 \\
\text { Hijo/otro }=4\end{array}$ & 6 meses & SF-36 & $\begin{array}{l}\text { Entrenamiento de } \\
\text { ejercicio físico en el } \\
\text { hogar, psicoeducación, } \\
\text { entrenamiento en } \\
\text { habilidades de } \\
\text { comunicación y } \\
\text { entrenamiento en } \\
\text { actividades placenteras } \\
\text { tanto en enfermos como } \\
\text { cuidadores. }\end{array}$ & $\begin{array}{l}\text { Este estudio no mostró ningún } \\
\text { efecto beneficioso. }\end{array}$ \\
\hline
\end{tabular}




\begin{tabular}{|c|c|c|c|c|c|c|c|}
\hline Primer autor y año & País & Idioma & Población & Periodo & $\begin{array}{l}\text { Método de evaluar la calidad } \\
\text { de vida }\end{array}$ & Intervención & Resultado principal \\
\hline Tanner et al., 2015(22) & EE.UU. & Inglés & $\begin{array}{l}\mathrm{N} \text { total }=278 \\
\mathrm{H} / \mathrm{M}=129 / 149 \\
\text { Edad media }=67,1 \\
\text { Cónyuge }=44 \\
\text { Hijo }=47 \\
\text { Otro }=9 \\
\mathrm{Gi}=100 \\
\mathrm{H} / \mathrm{M}=25 / 75 \\
\text { Edad media }=66,3 \\
\text { Cónyuge }=41 \\
\text { Hijo }=49 \\
\text { Otro }=10 \\
\mathrm{GC}=178 \\
\mathrm{H} / \mathrm{M}=104 / 74 \\
\text { Edad media }=67,5 \\
\text { Cónyuge }=46 \\
\text { Hijo }=46 \\
\text { Otro }=8\end{array}$ & 18 meses & $\begin{array}{l}\text {-Carga objetiva evaluada con } 3 \\
\text { preguntas cortas } \\
\text {-Carga subjetiva evaluada } \\
\text { mediante Zarit Burden Interview } \\
\text { (ZBI), } 12 \text { ítems } \\
\text {-Escala salud mental y física } \\
\text { (SF-12) }\end{array}$ & Breve guía de recursos. & $\begin{array}{l}\text { No se encontraron efectos } \\
\text { significativos, solo una modesta } \\
\text { reducción del tiempo de } \\
\text { dedicación de los cuidadores. }\end{array}$ \\
\hline $\begin{array}{l}\text { Xiao et al., } \\
2015^{(23)}\end{array}$ & Australia & Inglés & $\begin{array}{l}N \text { total }=61 \\
H / M=51 / 45 \\
\text { Edad media }=56,0 \\
\text { Gi }=31 \\
H / M=26 / 23 \\
\text { Edad media }=56,0 \\
G C=30 \\
H / M=25 / 22 \\
\text { Edad media }=56,0\end{array}$ & 12 meses & $\begin{array}{l}\text {-SF-36 } \\
\text {-Caregiver distress }\end{array}$ & $\begin{array}{l}\text { Diario del cuidador, } \\
\text { inventario de las } \\
\text { necesidades del cuidador, } \\
\text { reuniones mensuales, y } \\
\text { sesiones de información, } \\
\text { todo ello en el idioma } \\
\text { elegido por el cuidador. }\end{array}$ & $\begin{array}{l}\text { Aumento significativo de la calidad } \\
\text { de vida en comparación con la } \\
\text { atención habitual. }\end{array}$ \\
\hline Livingston et al., 2014(24) & Reino Unido & Inglés & $\begin{array}{l}\text { N total }=260 \\
\text { H/M }=82 / 178 \\
\text { Edad media }=59,05 \\
\text { Cónyuge/pareja }=109 \\
\text { Hijo/a }=113 \\
\text { Amigo/a }=6 \\
\text { Pareja del hijo/a }=12 \\
\text { Sobrino/a = } \\
\text { Nieto/a }=6 \\
\text { Hermano/a }=4 \\
\text { Otro }=2 \\
\text { Gi }=173 \\
\text { H/M }=57 / 116 \\
\text { Edad media }=62,0 \\
\text { GC }=87 \\
\text { I/M }=25 / 62 \\
\text { Edad media }=56,1\end{array}$ & 2 años & $\begin{array}{l}- \text {-HADS } \\
\text {-Qol-AD } \\
- \text { HSQ }\end{array}$ & Manuales de 8 sesiones & Mejora de la salud mental en Gi. \\
\hline
\end{tabular}




\begin{tabular}{|c|c|c|c|c|c|c|c|}
\hline Primer autor y año & País & Idioma & Población & Periodo & $\begin{array}{l}\text { Método de evaluar la calidad } \\
\text { de vida }\end{array}$ & Intervención & Resultado principal \\
\hline $\begin{array}{l}\text { Keeping-Burke et al., } \\
\left.2013^{25}\right)\end{array}$ & Canadá & Inglés & $\begin{array}{l}\text { N total = } 182 \\
\text { H/M }=\text { No consta } \\
\text { Edad media }=58,65 \\
\text { Gi }=91 \\
\text { H/M }=\text { No consta } \\
\text { Edad media }=59,1 \\
\text { GC }=91 \\
\text { H/M }=\text { No consta } \\
\text { Edad media }=58,2\end{array}$ & 1 año y 2 meses & $\begin{array}{l}\text {-Ansiedad (S-STAI) } \\
\text {-Sintomas depresivos (CESD-10) }\end{array}$ & Telesalud & $\begin{array}{l}\text { Disminución de ansiedad (en } \\
\text { cuidadores de pacientes varones) } \\
\text { y sítomas depresivos (en } \\
\text { cuidadores de ambos sexos). }\end{array}$ \\
\hline McMillan et al., 2011 & EE.UU. & Inglés & $\begin{array}{l}\text { N total }=709 \\
H / M=187 / 522 \\
\text { Edad media }=72,66 \\
\text { Gi }=371 \\
H / M=99 / 272 \\
\text { Edad media }=72,67 \\
G c=338 \\
H / M=88 / 250 \\
\text { Edad media }=72,65\end{array}$ & $\begin{array}{l}\text { Cuidadores que están } \\
\text { en el hospital al menos } \\
4 \text { horas al día (no } \\
\text { especifica el número de } \\
\text { días) }\end{array}$ & $\begin{array}{l}\text {-Forma abreviada de Boston } \\
\text { del Centro de Estudios } \\
\text { Epidemiológico-Depresión. } \\
\text {-Cuestionario corto del estado } \\
\text { mental (SPMSQ). }\end{array}$ & $\begin{array}{l}\text { Informes de datos } \\
\text { estandarizados. }\end{array}$ & $\begin{array}{l}\text { No se obtuvieron resultados } \\
\text { significativos. }\end{array}$ \\
\hline Czaja et al., 2009(27) & EE. UU. & Inglés & $\begin{array}{l}\text { Ntotal }=643 \\
\text { H/M }=38 / 182 \\
\text { Edad media }=60,6 \\
\text { n cuidador hispano }=212 \\
H / M=38 / 177 \\
\text { Edad media }=60,1 \\
\text { Cónyuge }=83 \\
\text { Hijo }=109 \\
\text { Hermano }=9 \\
\text { Otro }=11 \\
\text { n cuidador blanco = } 220 \\
H / M=38 / 182 \\
\text { Edad media }=63,9 \\
\text { Cónyuge }=126 \\
\text { Hijo }=86 \\
\text { Hermano }=2 \\
\text { Otro }=6 \\
\text { n cuidador negro }=211 \\
\text { H/M }=34 / 177 \\
\text { Edad media }=58,5 \\
\text { Cónyuge }=63 \\
\text { Hijo }=113 \\
\text { Hermano }=7 \\
\text { Otro }=28\end{array}$ & 6 meses & $\begin{array}{l}\text {-Entrevista sobre la carga del } \\
\text { cuidador } \\
\text {-Escala de Depresión del Centro } \\
\text { de Estudios } \\
\text { Epidemiológicos (CES-D) }\end{array}$ & Información de medidas & $\begin{array}{l}\text { Los hispanos que brindan } \\
\text { cuidados tenían un riesgo } \\
\text { levemente mayor de depresión que } \\
\text { los otros. } \\
\text { Los cuidadores afroamericanos e } \\
\text { hispanos informaron menos carga } \\
\text { que los cuidadores blancos. }\end{array}$ \\
\hline Wilson et al., 2009(28) & Reino Unido & Inglés & $\begin{array}{l}\text { N total }=236 \\
H / M=\text { no consta } \\
\text { Edad media = no consta }\end{array}$ & 24 meses & $\begin{array}{l}- \text { HADS } \\
-E Q-5 D\end{array}$ & $\begin{array}{l}\text { Visitas semanales } \\
\text { amistosas }\end{array}$ & $\begin{array}{l}\text { Tendencia no significativa a la } \\
\text { mejora de calidad de vida de los } \\
\text { cuidadores. }\end{array}$ \\
\hline
\end{tabular}




\begin{tabular}{|c|c|c|c|c|c|c|c|}
\hline Primer autor y año & País & Idioma & Población & Periodo & $\begin{array}{l}\text { Método de evaluar la calidad } \\
\text { de vida }\end{array}$ & Intervención & Resultado principal \\
\hline Guerrero et al., 2008 & España & Español & $\begin{array}{l}\mathrm{N} \text { total }=61 \\
H / M=12 / 49 \\
\mathrm{Edad} \text { media }=60,55 \\
\text { Gi }=29 \\
\mathrm{H} / \mathrm{M}=5 / 24 \\
\mathrm{Edad} \text { media }=60,0 \\
\mathrm{GC}=32 \\
\mathrm{H} / \mathrm{M}=7 / 25 \\
\text { Edad media }=61,1\end{array}$ & 1 año y 1 mes & $\begin{array}{l}\text {-Cuestionarios COOP/WONCA } \\
\text {-Escala de Goldberg }\end{array}$ & $\begin{array}{l}\text {-Dos visitas (visita de } \\
\text { historia clínica y visita de } \\
\text { soporte psicológico) } \\
\text {-Dos llamadas telefónicas } \\
\text { de apoyo. }\end{array}$ & $\begin{array}{l}\text { Mejora significativa de la calidad } \\
\text { de vida del cuidador en Gi. }\end{array}$ \\
\hline Crotty et al., 2008 & Australia & Inglés & $\begin{array}{l}N \text { total }=98 \\
\mathrm{Gi}=\text { no consta } \\
\mathrm{H} / \mathrm{M}=\text { no consta } \\
\mathrm{Edad} \text { media }=\text { no consta } \\
\mathrm{GC}=\text { no consta } \\
\mathrm{H} / \mathrm{M}=\text { no consta } \\
\text { Edad media }=\text { no consta }\end{array}$ & 1 año & $\begin{array}{l}\text {-Calidad de vida (SF-36) } \\
\text { - Índice de tensión del cuidador } \\
\text { (CSI) }\end{array}$ & Programa de rehabilitación & $\begin{array}{l}\text { No hubo diferencias en la calidad } \\
\text { de vida, en cambio, se experimentó } \\
\text { mayor tensión en Gc. }\end{array}$ \\
\hline Walsh et al., $2007^{(31)}$ & Reino Unido & Inglés & $\begin{array}{l}\text { N total }=271 \\
\text { H/M }=56 / \\
\text { Edad media }=56,3 \\
\text { Cónyuge }=172 \\
\text { Hijo/a }=67 \\
\text { Otro }=32 \\
\text { Gi }=137 \\
\text { H/M }=29 \\
\text { Edad media }=56,4 \\
\text { Cónyuge }=92 \\
\text { Hijo/a }=29 \\
\text { Otro }=16 \\
\text { Gc }=134 \\
\text { H/M }=27 \\
\text { Edad media }=56,1 \\
\text { Cónyuge }=80 \\
\text { Hijo/a }=38 \\
\text { Otro }=16\end{array}$ & 2 años y 3 meses & $\begin{array}{l}\text {-Cuestionario general de salud } \\
\text { (GHQ-28) } \\
\text {-Carga del cuidador Carer Strain } \\
\text { Index (CSI) y calidad de vida } \\
\text { CQOLC }\end{array}$ & Visitas semanales & No hubo resultados significativos \\
\hline Thomas et al., 2007(32) & Canadá & Inglés & $\begin{array}{l}\mathrm{N} \text { total }=520 \\
\mathrm{H} / \mathrm{M}=170 / 350 \\
\mathrm{Edad} \text { media }=80,6 \\
\mathrm{Gi}_{1}=175 \\
\mathrm{H} / \mathrm{M}=66 / 109 \\
\text { Edad media }=80,7 \\
\text { Cónyuge }=49 \\
\mathrm{Gi} \mathrm{i}_{2}=170 \\
\mathrm{H} / \mathrm{M}=47 / 123 \\
\mathrm{Edad} \text { media }=80,4 \\
\mathrm{Cónyuge}=47 \\
\mathrm{Gc}=175 \\
\mathrm{H} / \mathrm{M}=57 / 118 \\
\text { Edad media }=80,7 \\
\text { Cónyuge }=49\end{array}$ & 4 años & $\begin{array}{l}\text { Inventario de Carga de } \\
\text { Cuidadores (CBI) }\end{array}$ & $\begin{array}{l}\text { Evaluación funcional } \\
\text { y asesoramiento de } \\
\text { enfermos. }\end{array}$ & No hubo resultados significativos. \\
\hline
\end{tabular}




\begin{tabular}{|c|c|c|c|c|c|c|c|}
\hline Primer autor y año & País & Idioma & Población & Periodo & $\begin{array}{l}\text { Método de evaluar la calidad } \\
\text { de vida }\end{array}$ & Intervención & Resultado principal \\
\hline Björkdahl et al., 2007(33) & Suecia & Inglés & $\begin{array}{l}\mathrm{N} \text { total }=35 \\
\text { Cónyuge hombre = } 9 \\
\text { Cónyuge mujer }=24 \\
\text { Hijo/a }=2 \\
\mathrm{Gi}=18 \\
\text { Cónyuge hombre }=6 \\
\text { Cónyuge mujer }=12 \\
\text { Hijo/a }=0 \\
\mathrm{Gc}=17 \\
\text { Cónyuge hombre }=3 \\
\text { Cónyuge mujer }=12 \\
\text { Hijo/a }=2\end{array}$ & 1 año & $\begin{array}{l}\text { Escala de carga del cuidador } \\
\text { (escala CB) }\end{array}$ & $\begin{array}{l}\text { Rehabilitación en el hogar } \\
\text { frente rehabilitación } \\
\text { ambulatoria. }\end{array}$ & $\begin{array}{l}\text { Tendencia a una reducción de } \\
\text { carga en el Gi. }\end{array}$ \\
\hline McCullagh et al., 2005 & Reino Unido & Inglés & $\begin{array}{l}N \text { total }=232 \\
H / M=83 / 149 \\
\text { Edad media }=65,7 \\
\text { Cónyuge/pareja }=164 \\
\text { Hijo/a }=53 \\
\text { Pariente/amigo }=15\end{array}$ & 1 año & $\begin{array}{l}\text {-Carga del cuidador (CBS) } \\
\text {-Medidas de calidad de vida } \\
\text { (QOL): EQ-5D, EQ-VAS }\end{array}$ & Formación de cuidadores & $\begin{array}{l}\text { Los niveles de ansiedad, depresión } \\
\text { y carga de los cuidadores se } \\
\text { redujeron significativamente. }\end{array}$ \\
\hline Larson et al., 2005(35) & Suecia & Inglés & $\begin{array}{l}\mathrm{N} \text { total }=100 \text { (cónyuges) } \\
H / M=20 / 80 \\
\text { Edad media }=67,41 \\
\mathrm{Gi}=50 \\
\mathrm{H} / \mathrm{M}=12 / 38 \\
\text { Edad media }=68,16 \\
\mathrm{GC}=50 \\
\mathrm{H} / \mathrm{M}=8 / 42 \\
\text { Edad media }=66,66\end{array}$ & 1 año y 8 meses & $\begin{array}{l}\text {-Medidas de calidad de vida } \\
\text { (QOL): EQ-VAS } \\
\text {-Cuestionario de bienestar de } \\
\text { Bradley } \\
\text {-Estado de salud percibido } \\
\text { (EuroQoL). }\end{array}$ & $\begin{array}{l}\text { Programa de educación y } \\
\text { apoyo }\end{array}$ & $\begin{array}{l}\text { No se encontraron diferencias } \\
\text { significativas. Ligero efecto positivo } \\
\text { en relación con aquellos que } \\
\text { asistieron más a los programas. }\end{array}$ \\
\hline Provinciali et al., 2004 & Italia & Inglés & $\begin{array}{l}N \text { total }=75 \\
H / M=17 / 58 \\
\text { Edad media }=55,8 \\
\text { Gi }=38 \\
H / M=8 / 30 \\
\text { Edad media }=53,8 \\
\text { Hija }=18 \\
\text { Hijo }=7 \\
\text { Nuera }=10 \\
\text { Nieto/a }=3 \\
\text { Gc }=37 \\
H / M=9 / 28 \\
\text { Edad media }=57,8 \\
\text { Hija }=\text { no consta } \\
\text { Hijo }=\text { no consta } \\
\text { Nuera }=\text { no consta } \\
\text { Nieto/a }=\text { no consta }\end{array}$ & No consta & $\begin{array}{l}\text {-Medidas de Carga del Cuidador } \\
\text { de Montgomery (MCBM) } \\
\text {-Escala de estrés relativo (RSS) } \\
\text {-Escala de ansiedad de } \\
\text { autoevaluación de Zung (ZSAS) } \\
\text { - Escala de depresión de } \\
\text { autoevelualuación (ZSDS) } \\
\text { - versión corta del Índice de } \\
\text { satisfacción con la vida (LSIA) } \\
\text { para medir calidad de vida } \\
\text { autopercibida }\end{array}$ & $\begin{array}{l}\text { Recolección de datos sobre } \\
\text { bienestar psicológico. }\end{array}$ & $\begin{array}{l}\text { Mayor ansiedad en Gi. No hubo } \\
\text { diferencias significativas en } \\
\text { depresión y calidad de vida. }\end{array}$ \\
\hline
\end{tabular}




\begin{tabular}{|c|c|c|c|c|c|c|c|}
\hline Primer autor y año & País & Idioma & Población & Periodo & $\begin{array}{l}\text { Método de evaluar la calidad } \\
\text { de vida }\end{array}$ & Intervención & Resultado principal \\
\hline Pozzilli et al., 20044 & Italia & Inglés & $\begin{array}{l}\mathrm{N} \text { total }=123 \\
\mathrm{H} / \mathrm{M}=41 / 82 \\
\text { Edad media }=53 \\
\text { Cónyuge/pareja }=66 \\
\text { Padres }=26 \\
\text { Hijos/as }=14 \\
\text { Hermanos }=6 \\
\text { Otros }=11\end{array}$ & 1 año & $\begin{array}{l}\text { Perfil de } \\
\text { estados de ánimo (POMS) }\end{array}$ & Educación de cuidadores. & $\begin{array}{l}\text { Morbilidad } \\
\text { psicológica de los cuidadores no } \\
\text { cambió significativamente. }\end{array}$ \\
\hline Kalra et al., 2004 ${ }^{(38)}$ & Reino Unido & Inglés & $\begin{array}{l}N \text { total }=300 \\
H / M=160 / 140 \\
\text { Edad media }=76 \\
\text { Gi }=151 \\
H / M=86 / 65 \\
\text { Edad media }=76 \\
\text { GC }=149 \\
\mathrm{H} / \mathrm{M}=74 / 75 \\
\text { Edad media }=76\end{array}$ & 1 año & $\begin{array}{l}\text {-Escala de carga del cuidador. } \\
\text {-EuroQol, visual analog scale: } \\
\text { EQ-VAS } \\
\text {-Indice de actividades Frenchay } \\
\text { (FAl) } \\
\text {-Escala de ansiedad y depresión } \\
\text { hospitalaria: HADS }\end{array}$ & $\begin{array}{l}\text { Formación de los } \\
\text { cuidadores }\end{array}$ & $\begin{array}{l}\text { Mejora de carga y salud emocional } \\
\text { de los cuidadores. }\end{array}$ \\
\hline Patel et al., 2004 ${ }^{(39)}$ & Reino Unido & Inglés & $\begin{array}{l}\mathrm{N} \text { total }=300 \\
\mathrm{H} / \mathrm{M}=\text { no consta } \\
\text { Edad media }=\text { no consta }\end{array}$ & 1 año & $-E Q-5 D$ & $\begin{array}{l}\text { Formación del cuidador en } \\
\text { enfermería básica y } \\
\text { facilitación de técnicas de } \\
\text { cuidado personal }\end{array}$ & $\begin{array}{l}\text { Mejora de la calidad de vida del } \\
\text { cuidador. }\end{array}$ \\
\hline Carnevale et al., 2002 $2^{40}$ & EE. UU. & Inglés & $\begin{array}{l}\mathrm{N} \text { total }=27 \\
\mathrm{H} / \mathrm{M}=18 / 9 \\
\text { Edad media }=47,5 \\
\mathrm{Gc}=10 \\
\mathrm{H} / \mathrm{M}=8 / 2 \\
\text { Edad media }=45,8 \\
\mathrm{G} \text { módulo educativo }=8 \\
\mathrm{H} / \mathrm{M}=4 / 4 \\
\text { Edad media }=42,3 \\
\mathrm{G} \text { módulo educativo + conductual }=9 \\
\mathrm{H} / \mathrm{M}=6 / 3 \\
\text { Edad media }=54,4\end{array}$ & 2 años & $\begin{array}{l}\text {-Cuestionario sobre recursos y } \\
\text { estrés (QRS) } \\
\text {-Versión adaptada del Maslach } \\
\text { Burnout Inventory (MBI) }\end{array}$ & $\begin{array}{l}\text {-Módulo educativo y } \\
\text { conductual }\end{array}$ & $\begin{array}{l}\text { No se encuentran diferencias } \\
\text { significativas. }\end{array}$ \\
\hline Gunnell et al., 2000 & Reino Unido & Inglés & $\begin{array}{l}N \text { total }=133 \\
H / M=58 / 75 \\
E d a d \text { media }=65 \\
\text { Gi }=93 \\
H / M=32 / 61 \\
E d a d \text { media }=65 \\
\text { Gc }=40 \\
H / M=26 / 14 \\
\text { Edad media }=66\end{array}$ & 1 año y 3 meses & $\begin{array}{l}\text {-COOP-WONCA } \\
\text {-EuroQol EQ-5D }\end{array}$ & Cuestionarios por correo & $\begin{array}{l}\text { No hubo diferencias en ninguna } \\
\text { medida. }\end{array}$ \\
\hline
\end{tabular}




\begin{tabular}{|c|c|c|c|c|c|c|c|}
\hline Primer autor y año & País & Idioma & Población & Periodo & $\begin{array}{l}\text { Método de evaluar la calidad } \\
\text { de vida }\end{array}$ & Intervención & Resultado principal \\
\hline Mohide et al., 1990(42) & Canadá & Inglés & $\begin{array}{l}\mathrm{N} \text { total }=60 \\
\mathrm{H} / \mathrm{M}=17 / 43 \\
\mathrm{Edad} \text { media }=67,75 \\
\mathrm{Gi}=30 \\
\mathrm{H} / \mathrm{M}=9 / 21 \\
\mathrm{Edad} \text { media }=66,10 \\
\mathrm{GC}=30 \\
\mathrm{H} / \mathrm{M}=8 / 22 \\
\text { Edad media }=69,40\end{array}$ & 6 meses & $\begin{array}{l}\text {-CES-D } \\
\text {-STAI } \\
\text {-CQLI }\end{array}$ & $\begin{array}{l}\text { Visitas domiciliarias, alentar } \\
\text { la búsqueda de ayuda } \\
\text { médica en problemas } \\
\text { de salud desatendidos } \\
\text { educación, } 4 \text { horas de } \\
\text { descanso semanal y } \\
\text { reunión mensual optativa. }\end{array}$ & $\begin{array}{l}\text { Se han observado niveles altos de } \\
\text { ansiedad y depresión y ninguna } \\
\text { intervención redujo los niveles } \\
\text { de estos. No hubo diferencias } \\
\text { significativas entre los grupos. Al } \\
\text { final del ensayo Gi experimentó } \\
\text { una mejor calidad de vida. }\end{array}$ \\
\hline Lawton et al., $1989^{(43)}$ & EE. UU. & Inglés & $\begin{array}{l}\mathrm{N} \text { total }=632 \\
\mathrm{H} / \mathrm{M}=131 / 501 \\
\text { Edad media }=59,9 \\
\text { Cónyuge }=287 \\
\text { Hijo//a }=244 \\
\text { Suegro }=36 \\
\text { Hermano/a }=35 \\
\text { Amigo/a }=12 \\
\text { Otro }=43 \\
\mathrm{Gi}=317 \\
\mathrm{H} / \mathrm{M}=61 / 256 \\
\text { Edad media }=60,4 \\
\text { Cónyuge }=158 \\
\text { Hijo//a }=114 \\
\text { Suegro }=13 \\
\text { Hermano/a }=12 \\
\text { Amigo/a }=5 \\
\text { Otro }=15 \\
\mathrm{GC}=315 \\
\mathrm{H} / \mathrm{M}=70 / 245 \\
\text { Edad media }=59,4 \\
\text { Cónyuge }=127 \\
\text { Hijo/a }=130 \\
\text { Suegro }=23 \\
\text { Hermano/a }=23 \\
\text { Amigo/a }=7 \\
\text { Otro }=28\end{array}$ & 12 meses & $\begin{array}{l}\text {-CESD-20 } \\
\text {-Escala de carga y bienestar del } \\
\text { cuidador }\end{array}$ & Relevo del cuidado. & $\begin{array}{l}\text { Satisfacción muy alta, aunque no } \\
\text { haya mejorado la salud mental y } \\
\text { calidad de vida. }\end{array}$ \\
\hline
\end{tabular}

Gc: grupo control

Gi: grupo in 
Al evaluar la calidad de los artículos seleccionados para la revisión, mediante el cuestionario CONSORT, las puntuaciones oscilaron entre un mínimo de 9,5 y un máximo de 20,5 (sobre 21 ítems) con mediana igual a 16 (tabla 2).

Los 29 (100\%) ensayos clínicos incluidos en la presente revisión fueron controlados y aleatorizados. Por tanto, según los criterios de la Scottish Intercollegiate Guidelines Network (SIGN) esta revisión presentó evidencia «1+» (revisiones sistemáticas de ensayos clínicos aleatorizados con bajo riesgo de sesgos) con un grado de recomendación $\mathrm{A}$.

La filiación fue mayoritariamente estadounidense, con 9 ensayos ${ }^{(15-17,21,22,26,27,40,43)}$ y británica con 7 ensayos $(24,28,31,34,38,39,41)$, estando todos los trabajos aceptados en esta revisión redactados en inglés a excepción del trabajo de Guerrero et al. ${ }^{(29)}$, que lo estuvo en español.

El ensayo de McMillan et al. ${ }^{(26)}$, publicado en el año 2011, fue el que mayor población estudió con una $\mathrm{N}=709$ cuidadores, con una relación hombre/mujer de 187/522. En general, la mujer fue la persona dedicada al cuidado del enfermo, siendo el parentesco más observado el de cónyuge o pareja.

La edad media de los cuidadores/as, todos adultos, osciló entre un mínimo de 40,1 años del trabajo de Bilgin et al. ${ }^{(18)}$ y un máximo de 74,1 años en el trabajo de von Kanel et al. ${ }^{(16)}$.

El periodo de seguimiento de los estudios revisados presentó largos periodos, oscilando entre un mínimo de 4 a 8 meses $^{(17)}$ y un máximo de 4 años ${ }^{(15)}$.

\section{Evaluación de la calidad de vida}

La forma de medir el resultado de la intervención fue muy heterogénea, siendo los cuestionarios genéricos European Quality of Life-5 Dimensions (EQ-5D) (28,34,39,41) y el Short Form Health Survey 36 items (SF-36) $)^{(20,21,23,30)}$ los que más se utilizaron: en 4 ocasiones cada uno de ellos. Aunque, también fueron muy usados, en 3 ocasiones, otros cuestionarios como los específicos, Escala de Depresión de 20 ítems (CESD-20)(20,42,43) y la Escala de Depresión de 10 ítems (CESD-10) ${ }^{(17,25,27)}$ y el genérico Short Form Health Survey 12 ítems (SF-12) ${ }^{(18,22,28)}$. También, sería adecuado señalar la utilización de Escalas Analógicas Visuales para medir la calidad de vida de los/las cuidadores ${ }^{(34,35,38)}$.

Algunos ensayos utilizaron más una forma de evaluar la intervención. Así, los estudios de Provinciali et al. ${ }^{(36)}$ y de Kalra et al. ${ }^{(38)}$, usaron 4 métodos de medición. 


\begin{tabular}{|c|c|c|c|c|c|c|c|c|c|c|c|c|c|c|c|c|c|c|c|c|c|c|c|c|c|c|c|}
\hline & 1 & 2 & 3 & 4 & 5 & 6 & 7 & 8 & 9 & 10 & 11 & 12 & 13 & 14 & 15 & 16 & 17 & 18 & 19 & 20 & 21 & 22 & 23 & 24 & 25 & Total & Total (\%) \\
\hline $\begin{array}{l}\text { Demiris, } \\
\text { et al!(15) }\end{array}$ & 1 & 1 & 1 & 1 & 1 & 1 & 0,5 & 1 & NA & NA & NA & 1 & 1 & 1 & 1 & 1 & 1 & NA & 1 & 1 & 1 & 1 & 1 & 1 & 1 & 20,5 & $98 \%$ \\
\hline $\begin{array}{l}\text { von Kanel, } \\
\text { et al (16) }\end{array}$ & 0,5 & 1 & 1 & 1 & 1 & 0,5 & 0 & 1 & NA & NA & NA & 0 & 0 & 0 & 0 & 0 & 1 & NA & 0 & 1 & 1 & 1 & 1 & 0 & 1 & 12 & $57 \%$ \\
\hline $\begin{array}{l}\text { Gittin, } \\
\text { et al.117) }\end{array}$ & 1 & 1 & 1 & 0,5 & 1 & 1 & 0,5 & 1 & NA & NA & NA & 1 & 1 & 1 & 0 & 1 & 1 & NA & 1 & 1 & 1 & 1 & 1 & 1 & 1 & 19 & $90 \%$ \\
\hline $\begin{array}{l}\text { Bilgin, } \\
\text { et al. }{ }^{(18)}\end{array}$ & 0 & 1 & 1 & 1 & 1 & 1 & 0,5 & 1 & NA & NA & NA & 0 & 0 & 0 & 1 & 1 & 1 & NA & 1 & 1 & 1 & 1 & 0 & 0 & 1 & 14,5 & $69 \%$ \\
\hline $\begin{array}{l}\text { Brijoux, } \\
\text { et all.19) }\end{array}$ & 1 & 1 & 1 & 1 & 1 & 1 & 0,5 & 1 & NA & NA & NA & 1 & 1 & 1 & 1 & 1 & 1 & NA & 1 & 1 & 1 & 1 & 1 & 0 & 1 & 19,5 & $93 \%$ \\
\hline $\begin{array}{l}\text { Kuo, } \\
\text { et al. }{ }^{200}\end{array}$ & 0 & 1 & 1 & 1 & 1 & 1 & 0,5 & 1 & NA & NA & NA & 1 & 1 & 1 & 1 & 1 & 1 & NA & 1 & 1 & 1 & 1 & 0 & 0 & 1 & 17,5 & $83 \%$ \\
\hline $\begin{array}{l}\text { Prick, } \\
\text { et al. }{ }^{211)}\end{array}$ & 1 & 1 & 1 & 1 & 1 & 1 & 0,5 & 1 & NA & NA & NA & 1 & 1 & 1 & 1 & 1 & 1 & NA & 1 & 0 & 1 & 1 & 1 & 1 & 1 & 19,5 & $93 \%$ \\
\hline $\begin{array}{l}\text { Tanner, } \\
\text { et al. }{ }^{222)}\end{array}$ & 1 & 1 & 1 & 1 & 1 & 1 & 0,5 & 1 & NA & NA & NA & 1 & 1 & 1 & 1 & 1 & 1 & NA & 1 & 1 & 1 & 1 & 0 & 0 & 1 & 18,5 & $88 \%$ \\
\hline $\begin{array}{l}\text { Xiao, } \\
\text { et al. }{ }^{233)}\end{array}$ & 0 & 1 & 1 & 1 & 1 & 1 & 0,5 & 1 & NA & NA & NA & 1 & 0 & 1 & 1 & 1 & 1 & NA & 0 & 1 & 1 & 1 & 0 & 0 & 1 & 15,5 & $74 \%$ \\
\hline $\begin{array}{l}\text { Livingston, } \\
\text { et all. }{ }^{24)}\end{array}$ & 1 & 1 & 1 & 1 & 1 & 1 & 0,5 & 1 & NA & NA & NA & 1 & 1 & 1 & 1 & 1 & 1 & NA & 1 & 1 & 1 & 1 & 1 & 0 & 1 & 19,5 & $93 \%$ \\
\hline Keeping-Burke, et al.(.25) & 0 & 1 & 1 & 0,5 & 1 & 1 & 0,5 & 1 & NA & NA & NA & 1 & 1 & 1 & 1 & 1 & 1 & NA & 1 & 1 & 1 & 1 & 1 & 0 & 0 & 17 & $81 \%$ \\
\hline $\begin{array}{l}\text { McMillan, } \\
\text { et al.(26) }\end{array}$ & 0,5 & 1 & 0,5 & 0,5 & 1 & 0,5 & 0,5 & 0 & NA & NA & NA & 1 & 0 & 0 & 1 & 1 & 1 & NA & 1 & 0 & 1 & 1 & 0 & 0 & 1 & 12,5 & $60 \%$ \\
\hline $\begin{array}{l}\text { Czaja, } \\
\text { et al. }{ }^{277}\end{array}$ & 0,5 & 1 & 0,5 & 0,5 & 1 & 0,5 & 0,5 & 0 & NA & NA & NA & 0 & 0 & 1 & 1 & 1 & 1 & NA & 1 & 0 & 1 & 1 & 0 & 0 & 1 & 12,5 & $60 \%$ \\
\hline $\begin{array}{l}\text { Wilson, } \\
\text { et al. }{ }^{288)}\end{array}$ & 0,5 & 1 & 0,5 & 1 & 1 & 0,5 & 0 & 0 & NA & NA & NA & 0 & 0 & 1 & 0 & 0 & 1 & NA & 0 & 0 & 1 & 1 & 0 & 0 & 1 & 9,5 & $45 \%$ \\
\hline $\begin{array}{l}\text { Guerrero, } \\
\text { et al.(20) }\end{array}$ & 0,5 & 1 & 0,5 & 1 & 1 & 1 & 0,5 & 1 & NA & NA & NA & 1 & 0 & 1 & 1 & 1 & 1 & NA & 1 & 1 & 1 & 1 & 0 & 0 & 1 & 16,5 & $79 \%$ \\
\hline $\begin{array}{l}\text { Crotty, } \\
\text { et all. }{ }^{30)}\end{array}$ & 1 & 1 & 0,5 & 1 & 1 & 1 & 0,5 & 1 & NA & NA & NA & 1 & 1 & 1 & 0 & 0 & 1 & NA & 1 & 1 & 1 & 1 & 1 & 0 & 1 & 17 & $81 \%$ \\
\hline
\end{tabular}




\begin{tabular}{|c|c|c|c|c|c|c|c|c|c|c|c|c|c|c|c|c|c|c|c|c|c|c|c|c|c|c|c|}
\hline & 1 & 2 & 3 & 4 & 5 & 6 & 7 & 8 & 9 & 10 & 11 & 12 & 13 & 14 & 15 & 16 & 17 & 18 & 19 & 20 & 21 & 22 & 23 & 24 & 25 & Total & Total $(\%)$ \\
\hline $\begin{array}{l}\text { Walsh, } \\
\text { et al.(31) }\end{array}$ & 0,5 & 1 & 1 & 0,5 & 1 & 1 & 0,5 & 1 & NA & NA & NA & 1 & 1 & 1 & 1 & 1 & 1 & NA & 1 & 1 & 1 & 1 & 0 & 0 & 1 & 16,5 & $79 \%$ \\
\hline $\begin{array}{l}\text { Thomas, } \\
\text { et al! }{ }^{(32)}\end{array}$ & 1 & 1 & 1 & 0,5 & 1 & 1 & 1 & 1 & NA & NA & NA & 1 & 1 & 1 & 1 & 1 & 1 & NA & 1 & 0 & 1 & 1 & 1 & 0 & 0 & 17,5 & $83 \%$ \\
\hline $\begin{array}{l}\text { Björkdahl, } \\
\text { et al. }{ }^{(33)}\end{array}$ & 0,5 & 1 & 0,5 & 0,5 & 1 & 0,5 & 1 & 1 & NA & NA & NA & 0 & 0 & 1 & 0 & 1 & 1 & NA & 0 & 1 & 1 & 1 & 0 & 0 & 1 & 13 & $62 \%$ \\
\hline $\begin{array}{l}\text { McCullagh, } \\
\text { et al. }{ }^{(.4)}\end{array}$ & 0,5 & 1 & 1 & 0,5 & 1 & 0,5 & 0 & 1 & NA & NA & NA & 1 & 0 & 1 & 0 & 1 & 1 & NA & 0 & 1 & 1 & 1 & 0 & 0 & 1 & 13,5 & $64 \%$ \\
\hline $\begin{array}{l}\text { Larson, } \\
\text { et al.(.35) }\end{array}$ & 1 & 1 & 0,5 & 1 & 1 & 1 & 0,5 & 1 & NA & NA & NA & 1 & 1 & 1 & 1 & 1 & 1 & NA & 1 & 0 & 1 & 1 & 0 & 0 & 0 & 16 & $76 \%$ \\
\hline $\begin{array}{l}\text { Provinciali, } \\
\text { et al. }{ }^{36)}\end{array}$ & 0,5 & 1 & 0,5 & 1 & 1 & 0,5 & 0 & 1 & NA & NA & NA & 1 & 0 & 0 & 1 & 1 & 1 & NA & 0 & 1 & 1 & 1 & 0 & 0 & 1 & 13,5 & $64 \%$ \\
\hline $\begin{array}{l}\text { Pozzilli, } \\
\text { et al.(37) }\end{array}$ & 0 & 1 & 1 & 1 & 1 & 0,5 & 0 & 1 & NA & NA & NA & 1 & 0 & 1 & 0 & 0 & 1 & NA & 0 & 0 & 1 & 1 & 0 & 0 & 1 & 11,5 & $55 \%$ \\
\hline $\begin{array}{l}\text { Kalra, } \\
\text { et al.(.38) }\end{array}$ & 1 & 1 & 0 & 0,5 & 1 & 1 & 0,5 & 1 & NA & NA & NA & 1 & 1 & 1 & 1 & 1 & 1 & NA & 1 & 1 & 1 & 1 & 0 & 0 & 1 & 17 & $81 \%$ \\
\hline $\begin{array}{l}\text { Patel, } \\
\text { et al.(39) }\end{array}$ & 0,5 & 1 & 0 & 1 & 1 & 0,5 & 1 & 1 & NA & NA & NA & 1 & 0 & 1 & 0 & 0 & 1 & NA & 0 & 1 & 1 & 1 & 0 & 0 & 0 & 12 & $57 \%$ \\
\hline $\begin{array}{l}\text { Carnevale, } \\
\left.\text { et al! }{ }^{400}\right)\end{array}$ & 0,5 & 1 & 1 & 1 & 1 & 0,5 & 1 & 1 & NA & NA & NA & 1 & 0 & 1 & 1 & 1 & 1 & NA & 0 & 1 & 1 & 1 & 0 & 0 & 1 & 16 & $76 \%$ \\
\hline $\begin{array}{l}\text { Gunnell, } \\
\text { et al. }\left({ }^{4}\right)\end{array}$ & 1 & 1 & 1 & 1 & 1 & 0,5 & 1 & 1 & NA & NA & NA & 0 & 0 & 1 & 1 & 1 & 1 & NA & 0 & 0 & 1 & 1 & 0 & 0 & 1 & 14,5 & $69 \%$ \\
\hline $\begin{array}{l}\text { Mohide, } \\
\text { et al. }{ }^{(42)}\end{array}$ & 0,5 & 1 & 1 & 1 & 1 & 1 & 0,5 & 1 & NA & NA & NA & 0 & 0 & 1 & 1 & 1 & 1 & NA & 1 & 0 & 1 & 1 & 0 & 0 & 1 & 15 & $71 \%$ \\
\hline $\begin{array}{l}\text { Lawton, } \\
\left.\text { et al. }{ }^{(43)}\right)\end{array}$ & 0,5 & 1 & 0 & 1 & 1 & 0,5 & 0,5 & 1 & NA & NA & NA & 1 & 0 & 1 & 1 & 1 & 1 & NA & 0 & 0 & 1 & 1 & 0 & 0 & 0 & 12,5 & $60 \%$ \\
\hline
\end{tabular}




\section{Intervención realizada}

La intervención que se realizó con una mayor frecuencia fue la formación de los cuidadores, en 10 ensayos, ${ }^{(19,20,23,34,35,37-40,42)}$. Aunque, también serían destacable las visitas domiciliarias de apoyo al cuidador/a que se realizaron en 3 ensayos ${ }^{(18,31,42)}$.

De todos modos, y en línea con los diferentes métodos de evaluación vistos anteriormente, también la forma de intervenir fue muy heterogénea, identificándose hasta 32 formas diferentes.

\section{Principales resultados observados}

Las diferentes intervenciones realizadas Ilevaron a que en 7 de los estudios ${ }^{(18-20,23,29,39,42)}$, se encontrase una mejoría significativa en la calidad de vida de los cuidadores/as.

También, el trabajo de Lawton et al. ${ }^{(43)}$, demostró una alta satisfacción, sin una clara mejora de la salud mental y de la calidad de vida del cuidador/a; 2 ensayos manifestaron una mejora de la salud

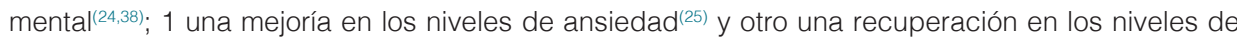
ansiedad y depresión ${ }^{(34)}$.

Asimismo, en 5 ensayos clínicos la intervención realizada redujo la carga del cuidador, sin un claro resultado, en el grupo intervención frente al control (19,30,33,34,38).

Por otro lado, 12 de los ensayos clínicos recogidos en esta revisión no mostraron ningún resultado, o diferencia significativa, en relación con la calidad de vida entre el grupo de la intervención y el contro| ${ }^{21,22,26,28,30-32,35-37,40,41)}$.

\section{DISCUSIÓN}

Los resultados observados, en esta revisión, llevan a deducir, aunque no en su totalidad, que la ayuda al cuidador/a puede llevar a mejorar su calidad de vida o al menos a sentir una mejoría en su salud mental.

De acuerdo con las recomendaciones sobre los objetivos de una revisión sistemática ${ }^{(44)}$, la actual tenía como finalidad sintetizar la información relevante relacionada con las intervenciones realizadas en los cuidadores de las personas adultas en ingreso domiciliario y poner a disposición de la comunidad científica información importante que pueda ayudar a comparar los diferentes programas existentes.

El análisis de la actualidad/obsolescencia de los estudios incluidos presentó unos resultados ligeramente más actuales a los obtenidos en estudios bibliométricos anteriores sobre hospitalización a domicilio ${ }^{(45,46)}$.

La evaluación de la calidad de los estudios incluidos en este trabajo mediante CONSORT dio unos resultados muy adecuados y superiores a los observados en otras revisiones similares ${ }^{(5,9)}$. El estudio de la progresión en la calidad documental que se observó en los ensayos más actuales se debe fundamentalmente a la implantación de estos cuestionarios. De hecho, los trabajos más antiguos no solían seguir estas guías de calidad ya que su aparición no fue hasta el año 1996 y su utilización fue de forma progresiva ${ }^{(47)}$.

El diseño de los estudios revisados mostró una alta evidencia al tratarse de ensayos clínicos aleatorizados, según los criterios de la Scottish Intercollegiate Guidelines Network (SIGN) ${ }^{(14)}$. El circunscribir la revisión a ensayos clínicos se debió a la búsqueda de una consistente relación causaefecto, ya que se buscaban trabajos de intervención ${ }^{(48)}$. Esta clasificación se basa en los diseños de 
los estudios de donde proviene la evidencia, asumiendo que algunos de ellos están sujetos a más sesgos que otros y, por ende, justifican más débilmente las decisiones clínicas ${ }^{(49)}$.

Era predecible la filiación anglófona de una mayoría de artículos y que estuvieran redactados, principalmente, en lengua inglesa. Este idioma es el elegido para la publicación de la mayoría de los artículos ya que hacerlo en otra lengua distinta resulta negativo para la visibilidad y análisis de citas. Asimismo, el número de revistas anglófonas contenidas en las principales bases de datos bibliográficas es muy elevado y publicar en ellas facilita la citación $n^{(5)}$.

El rango de edad de las personas cuidadoras, principalmente mujeres, como figura predominante en los ensayos revisados era previsible. El aumento de la cronicidad de las enfermedades y de las personas dependientes como consecuencia del envejecimiento de la población es un hecho demostrado, como también lo es que las mujeres son las principales implicadas en el cuidado familiar(50). En consecuencia, la edad de la cuidadora principal se debe relacionar con el parentesco, generalmente la pareja/esposa del paciente(6).

En cuanto al tiempo de cuidado, predominan las cuidadoras con un tiempo de experiencia de más de 18 meses y una dedicación de más de 12 horas diarias, que se desempeñan, además, como únicos cuidadores ${ }^{(51)}$, situación también observada en esta revisión y que avala la adecuación del periodo de estudio constatado en los ensayos analizados. De acuerdo con lo publicado por Brody ${ }^{(52)}$, debido al incremento de la esperanza de vida y de las enfermedades crónicas, la familia se vio obligada a "inventar" el cuidado de largo plazo.

La calidad de vida es un concepto que ha ido evolucionando a lo largo de los años, a pesar de ello no hay una definición universal. Existen diversos instrumentos para medirla. La necesidad de ellos para establecer el grado de calidad de vida en las personas es esencial en muchas disciplinas $^{(53)}$.

Las diferentes formas de evaluar los resultados de la intervención, en este caso la calidad de vida, y la heterogeneidad de los cuestionarios utilizados también ha sido observada en otros estudios anteriores ${ }^{(2)}$. Elegir una medida adecuada y utilizarla en la práctica clínica puede ser problemático. Sin embargo, es fundamental que las personas sean capaces de evaluar su propia calidad de vida ${ }^{(54)}$.

Diversos cuestionarios de calidad de vida, tanto genéricos como específicos, han demostrado que reúnen las propiedades psicométricas de fiabilidad, validez y sensibilidad al cambio y, por tanto, son aptos para estudios de tipo descriptivo y/o evaluativo en diferentes situaciones. Los cuestionarios específicos, a diferencia de los genéricos, que son aplicables a todo tipo de personas y poblaciones, incluida la población general, fueron diseñados a partir de los síntomas, las limitaciones y los trastornos de la vida diaria que producen una patología en concreto, con el objetivo de optimizar las propiedades del instrumento y, muy especialmente, la sensibilidad al cambio. No obstante, la teórica superioridad de unos cuestionarios respecto a otros debe probarse mediante estudios que comparen los resultados obtenidos entre los diferentes instrumentos administrados conjuntamente a un mismo grupo de personas ${ }^{(55)}$.

La formación, principal intervención observada en esta revisión, es imprescindible en los ámbitos del cuidado, no solo favorece tener mejores estrategias de afrontamiento y entender mejor las situaciones, sino que también ayuda a mejorar la calidad de vida tanto de los cuidadores como de las personas a su cargo.

Hay un acuerdo en considerar la formación como elemento importante a la hora de aplicar unos cuidados de calidad a las personas que atienden. Ello pone de manifiesto que es necesario tener una formación, a poder ser reglada, para el cuidado de personas en ingreso domiciliario. Sin embargo, hay la tendencia a pensar que existen situaciones que se solventan por el hecho de ser mujer y tener socializado el cuidar ${ }^{(6)}$. El cuidador/a bien formado es el principal apoyo de los servicios 
sanitarios domiciliarios y hay que tener en cuenta que, en determinados casos, gran parte de los cuidados que precisa el paciente son proporcionados por cuidadores no sanitarios ${ }^{(8)}$.

Las diferentes intervenciones realizadas mostraron que formar y ayudar al cuidador/a, expusieron una mejoría en su la calidad de vida. Y, también señalaron una mejoría en sus niveles de ansiedad.

Ser cuidador no es tarea fácil, porque exige, en general, asumir unas responsabilidades y unas tareas muy pesadas. Por eso, es importante evitar caer en una rutina de desgaste físico y psicológico que acabe pasando factura. Constituye el llamado "síndrome del cuidador": un trastorno caracterizado por un gradual agotamiento físico y psíquico que se presenta en personas que desempeñan el rol de cuidador principal de una persona dependiente. Así, el cuidador/a debe aprender a cuidarse física y emocionalmente(56).

La calidad de vida, junto con la medida del nivel de salud, se han reconocido como el objetivo central de la asistencia sanitaria, lo que ha subrayado la importancia de lo que podríamos denominar «medidas centradas en la persona» ${ }^{(57)}$. El progresivo aumento de la esperanza de vida de la población y el incremento de las patologías crónicas han hecho que el esfuerzo terapéutico deba centrarse en lograr una mejoría en la sensación de bienestar y satisfacción percibidas por el paciente, ya que lo importante no es tanto «estar bien» como «sentirse bien»(58).

\section{Limitaciones de este estudio:}

Quizá el haber ceñido la búsqueda a las tres principales bases de datos de las ciencias de la salud (que cuentan con un lenguaje normalizado de indización) pueda considerarse una limitación. Ahora bien, dado el buen número de ensayos clínicos incluidos en esta revisión, la búsqueda de la mayor pertinencia compense el haber optado por unas condiciones más restrictivas. Existen revisiones sistemáticas previas que consideran que interrogar bases de datos sin una adecuada indización aporta "ruido" a los resultados(59,60).

Si se consideró un importante inconveniente el alto número de ensayos que no pudieron ser admitidos al no adecuarse a los criterios establecidos ya que no existió una clara relación causal entre la intervención y el resultado basado en la evaluación de la calidad de vida: en unas ocasiones por medirlo junto a las personas cuidadas (sin diferenciarlos) en otras por no haber una clara diferenciación entre lo observado en relación con la CVRS y otras observaciones.

\section{Por todo lo observado, se podría concluir:}

Formar y ayudar al cuidador/a supuso una mejoría en su la calidad de vida y en la mejoría de los niveles de ansiedad. Aunque, en algunos ensayos no se encontraron diferencias entre el grupo de la intervención y el grupo control.

El hecho de intervenir (preocuparse del cuidador/a) supuso una menor carga en el estado anímico del mismo, aunque sin resultados claramente significativos.

\section{BIBLIOGRAFÍA}

1. Hendry F, McVittie C. Is quality of life a healthy concept? Measuring and understanding life experiences of older people. Qual Health Res. 2004;14(7):961-75. DOI: 10.1177/1049732304266738 
2. Wanden-Berghe C, Sanz-Valero J, Escribà-Agüir V, Castelló-Botia I, Guardiola-Wanden-Berghe R, Red de Malnutrición en Iberoamérica - Ciencia y Tecnología para Desarrollo (Red Mel CYTED). Evaluation of quality of life related to nutritional status. Br J Nutr. 2009;101(7):950-60. DOI: 10.1017/S0007114508207178

3. Wanden-Berghe C, Nolasco A, Sanz-Valero J, Planas M, Cuerda C, Group NADYA-SENPE. Health-related quality of life in patients with home nutritional support. J Hum Nutr Diet. 2009;22(3):219-25. DOI: 10.1111/j.1365-277x.2009.00957.x

4. Wanden-Berghe C, Martín-Rodero H, Guardiola-Wanden-Berghe R, Sanz-Valero J, GalindoVillardón P. Quality of life questionnaire related to nutritional status. Nutr Hosp. 2012;27(6):187685. DOI: 10.3305/nh.2012.27.6.6025

5. Martínez-González R, Sanz-Valero J, Wanden-Berghe C. Calidad de vida de las personas adultas con nutrición parenteral ingresadas en hospitalización domiciliaria: revisión sistemática. Hosp Domic. 2020;4(3):117-31. DOI: 10.22585/hospdomic.v4i3.109

6. Wanden-Berghe C, Nolasco A, Planas M, Sanz-Valero J, Rodríguez T, Cuerda C, et al. Healthrelated quality of life according to the main caregiver in patients with home nutritional support. Med Clin (Barc). 2008;131(8):281-4. DOI: 10.1016/s0025-7753(08)72258-9

7. Gomes B, Calanzani N, Curiale V, McCrone P, Higginson IJ. Effectiveness and cost-effectiveness of home palliative care services for adults with advanced illness and their caregivers. Cochrane Database Syst Rev. 2013;(6):CD007760. DOI: 10.1002/14651858.CD007760.pub2

8. Estrada Cuxart O, Massa Domínguez B, Ponce González MÁ, Mirón Rubio M, Torres Corts A, Mujal Martínez A, et al. Proyecto HAD 2020: una propuesta para consolidar la hospitalización a domicilio en España. Hosp Domic. 2017;1(2):93-117. DOI: 10.22585/hospdomic.v1i2.13

9. Álvarez Velásquez S, Sanz Valero J. Ventajas de la quimioterapia domiciliaria en los enfermos adultos con neoplasias: revisión sistemática. Hosp Domic. 2020;4(1):25-39. DI: 10.22585/hospdomic.v4i1.98

10. Donnelly N-A, Hickey A, Burns A, Murphy P, Doyle F. Systematic review and meta-analysis of the impact of carer stress on subsequent institutionalisation of community-dwelling older people. PloS One. 2015;10(6):e0128213. DOI: 10.1371/journal.pone.0128213

11. Crespo López M, López Martínez J. El apoyo a los cuidadores de familiares mayores dependientes en el hogar: desarrollo del programa "Cómo mantener su bienestar". Madrid, España: Instituto de Mayores y Servicios Sociales (IMSERSO); 2007.

12. Covarrubias Delgado L, Andrade Cepeda RMG. Calidad de Vida de Cuidadores de Pacientes Hospitalizados, nivel de dependencia y red de apoyo. Index Enferm. 2012;21(3):131-5. DOI: 10.4321/S1132-12962012000200005

13. Cobos-Carbó A, Augustovski F. CONSORT 2010 Declaration: updated guideline for reporting parallel group randomised trials. Med Clin (Barc). 2011;137(5):213-5. DOI: 10.1016/j.medcli.2010.09.034

14. Harbour R, Miller J. A new system for grading recommendations in evidence based guidelines. BMJ. 2001;323(7308):334-6. DOI: 10.1136/bmj.323.7308.334

15. Demiris G, Oliver DP, Washington K, Pike K. A Problem-Solving Intervention for Hospice Family Caregivers: A Randomized Clinical Trial. J Am Geriatr Soc. 2019;67(7):1345-52. DOI: 10.1111/ jgs. 15894 
16. von Känel R, Mausbach BT, Dimsdale JE, Ziegler MG, Mills PJ, Allison MA, et al. Refining caregiver vulnerability for clinical practice: determinants of self-rated health in spousal dementia caregivers. BMC Geriatr. 2019;19(1):18. DOI: 10.1186/s12877-019-1033-2

17. Gitlin LN, Arthur P, Piersol C, Hessels V, Wu SS, Dai Y, et al. Targeting Behavioral Symptoms and Functional Decline in Dementia: A Randomized Clinical Trial. J Am Geriatr Soc. 2018;66(2):33945. DOI: 10.1111/jgs. 15194

18. Bilgin S, Gozum S. Effect of nursing care given at home on the quality of life of patients with stomach cancer and their family caregivers' nursing care. Eur $J$ Cancer Care (Engl). 2018;27(2):e12567. DOI: 10.1111/ecc.12567

19. Brijoux T, Kricheldorff C, H LI M, Bonfico S. Supporting Families Living With Dementia in Rural Areas. Dtsch Arzteblatt Int. 2016;113(41):681-7. DOI: 10.3238/arztebl.2016.0681

20. Kuo L-M, Huang H-L, Hsu W-C, Wang Y-T, Shyu Y-IL. Home-based caregiver training: Benefits differ by care receivers' dementia diagnosis. Geriatr Nurs N Y N. 2016;37(5):376-84. DOI: 10.1016/j.gerinurse.2016.05.005

21. Prick A-E, de Lange J, Scherder E, Twisk J, Pot AM. The effects of a multicomponent dyadic intervention on the mood, behavior, and physical health of people with dementia: a randomized controlled trial. Clin Interv Aging. 2016;11:383-95. DOI: 10.2147/CIA.S95789

22. Tanner JA, Black BS, Johnston D, Hess E, Leoutsakos J-M, Gitlin LN, et al. A randomized controlled trial of a community-based dementia care coordination intervention: effects of MIND at Home on caregiver outcomes. Am J Geriatr Psychiatry Off J Am Assoc Geriatr Psychiatry. 2015;23(4):391-402. DOI: 10.1016/j.jagp.2014.08.002

23. Xiao LD, De Bellis A, Kyriazopoulos H, Draper B, Ullah S. The Effect of a Personalized Dementia Care Intervention for Caregivers From Australian Minority Groups. Am J Alzheimers Dis Dementiasr. 2016;31(1):57-67. DOI: 10.1177/1533317515578256

24. Livingston G, Barber J, Rapaport P, Knapp M, Griffin M, Romeo R, et al. START (STrAtegies for RelaTives) study: a pragmatic randomised controlled trial to determine the clinical effectiveness and cost-effectiveness of a manual-based coping strategy programme in promoting the mental health of carers of people with dementia. Health Technol Assess. 2014;18(61):1-242. DOI: 10.3310/hta18610

25. Keeping-Burke L, Purden M, Frasure-Smith N, Cossette S, McCarthy F, Amsel R. Bridging the transition from hospital to home: effects of the VITAL telehealth program on recovery for CABG surgery patients and their caregivers. Res Nurs Health. 2013;36(6):540-53. DOI: 10.1002/ nur.21571

26. McMillan SC, Small BJ, Haley WE. Improving hospice outcomes through systematic assessment: a clinical trial. Cancer Nurs. 2011;34(2):89-97. DOI: 10.1097/NCC.0b013e3181f70aee

27. Czaja SJ, Gitlin LN, Schulz R, Zhang S, Burgio LD, Stevens AB, et al. Development of the risk appraisal measure: a brief screen to identify risk areas and guide interventions for dementia caregivers. J Am Geriatr Soc. 2009;57(6):1064-72. DOI: 10.1111/j.1532-5415.2009.02260.x

28. Wilson E, Thalanany M, Shepstone L, Charlesworth G, Poland F, Harvey I, et al. Befriending carers of people with dementia: a cost utility analysis. Int J Geriatr Psychiatry. 2009;24(6):610-23. DOI: 10.1002/gps.2164

29. Guerrero Caballero L, Ramos Blanes R, Alcolado Aranda A, López Dolcet MJ, Pons La Laguna JL, Quesada Sabaté M. Multidisciplinary intervention program for caregivers of patients in a home care program. Gac Sanit. 2008;22(5):457-60. DOI: 10.1157/13126927 
30. Crotty M, Giles LC, Halbert J, Harding J, Miller M. Home versus day rehabilitation: a randomised controlled trial. Age Ageing. 2008;37(6):628-33. DOI: 10.1093/ageing/afn141

31. Walsh K, Jones L, Tookman A, Mason C, McLoughlin J, Blizard R, et al. Reducing emotional distress in people caring for patients receiving specialist palliative care. Randomised trial. $\mathrm{Br} J$ Psychiatry J Ment Sci. 2007;190:142-7. DOI: 10.1192/bjp.bp.106.023960

32. Thomas R, Worrall G, Elgar F, Knight J. Can they keep going on their own? A four-year randomized trial of functional assessments of community residents. Can J Aging Rev Can Vieil. 2007;26(4):379-90. DOI: 10.3138/cja.26.4.379

33. Björkdahl A, Nilsson AL, Sunnerhagen KS. Can rehabilitation in the home setting reduce the burden of care for the next-of-kin of stroke victims? J Rehabil Med. 2007;39(1):27-32. DOI: 10.2340/16501977-0001

34. McCullagh E, Brigstocke G, Donaldson N, Kalra L. Determinants of caregiving burden and quality of life in caregivers of stroke patients. Stroke. 2005;36(10):2181-6. DOI: 10.1161/01. STR.0000181755.23914.53

35. Larson J, Franzén-Dahlin A, Billing E, Arbin M, Murray V, Wredling R. The impact of a nurse-led support and education programme for spouses of stroke patients: a randomized controlled trial. J Clin Nurs. 2005;14(8):995-1003. DOI: 10.1111/j.1365-2702.2005.01206.x

36. Provinciali M, Moresi R, Muzzioli M, Tarabelli D, Sirolla C, Melchiorre MG, et al. Psychological, neuroendocrine and immune measures in non spousal carers of disabled elderly in Italy. Neuro Endocrinol Lett. 2004;25(5):391-6.

37. Pozzilli C, Palmisano L, Mainero C, Tomassini V, Marinelli F, Ristori G, et al. Relationship between emotional distress in caregivers and health status in persons with multiple sclerosis. Mult Scler Houndmills Basingstoke Engl. 2004;10(4):442-6. DOI: 10.1191/1352458504ms1046oa

38. Kalra L, Evans A, Perez I, Melbourn A, Patel A, Knapp M, et al. Training carers of stroke patients: randomised controlled trial. BMJ. 2004;328(7448):1099. DOI: 10.1136/bmj.328.7448.1099

39. Patel A, Knapp M, Evans A, Perez I, Kalra L. Training care givers of stroke patients: economic evaluation. BMJ. 2004;328(7448):1102. DOI: 10.1136/bmj.328.7448.1102

40. Carnevale GJ, Anselmi V, Busichio K, Millis SR. Changes in ratings of caregiver burden following a community-based behavior management program for persons with traumatic brain injury. $J$ Head Trauma Rehabil. 2002;17(2):83-95. DOI: 10.1097/00001199-200204000-00002

41. Gunnell D, Coast J, Richards SH, Peters TJ, Pounsford JC, Darlow MA. How great a burden does early discharge to hospital-at-home impose on carers? A randomized controlled trial. Age Ageing. 2000;29(2):137-42. DOI: 10.1093/ageing/29.2.137

42. Mohide EA, Pringle DM, Streiner DL, Gilbert JR, Muir G, Tew M. A randomized trial of family caregiver support in the home management of dementia. J Am Geriatr Soc. 1990;38(4):446-54. DOI: 10.1111/j.1532-5415.1990.tb03544.x

43. Lawton MP, Brody EM, Saperstein AR. A controlled study of respite service for caregivers of Alzheimer's patients. The Gerontologist. 1989;29(1):8-16. DOI: 10.1093/geront/29.1.8

44. Hagger MS. What makes a 'good' review article? Some reflections and recommendations. Health Psychol Rev. 2012;6(2):141-6. DOI: 10.1080/17437199.2012.705556

45. Domingo-Pueyo A. Análisis bibliométrico de la producción científica española, indizada en MEDLINE, sobre servicios de atención a domicilio provisto por hospital. Hosp Domic. 2017;1(3):14151. DOI: 10.22585/hospdomic.v1i3.24 
46. Sanz-Valero J, Wanden-Berghe C. Análisis bibliométrico de la producción científica, indizada en MEDLINE, sobre los servicios de salud proporcionados por las unidades de hospitalización a domicilio. Hosp Domic. 2017;1(1):21-34. DOI: 10.22585/hospdomic.v1i1.3

47. Begg C. Improving the quality of reporting of randomized controlled trials. The CONSORT statement. JAMA J Am Med Assoc. 1996;276(8):637-9. DOI: 10.1001/jama.276.8.637

48. Aracil-Lavado E, Wanden-Berghe C, Sanz-Valero J. Evaluación de la calidad de vida según el estado nutricional del paciente paliativo adulto: revisión sistemática. Hosp Domic. 2017;1(4):199210. DOI: 10.22585/hospdomic.v1i4.27

49. Manterola C, Asenjo-Lobos C, Otzen T. Jerarquización de la evidencia: Niveles de evidencia y grados de recomendación de uso actual. Rev Chil Infectologia. 2014;31(6):705-18. DOI: 10.4067/S0716-10182014000600011

50. Jiménez Ruiz I, Moya Nicolás M. La cuidadora familiar: sentimiento de obligación naturalizado de la mujer a la hora de cuidar. Enferm Glob. 2017;17(1):420-33. DOI: 10.6018/eglobal.17.1.292331

51. Achury DM, Castaño Riaño HM, Gómez Rubiano LA, Guevara Rodríguez NM. Calidad de vida de los cuidadores de pacientes con enfermedades crónicas con parcial dependencia. Investig En Enferm Imagen Desarro. 2011;13(1):27-46.

52. Brody EM. Prospects for family caregiving: Response to change, continuity, and diversity. En: Kane RA, Penrod JD, editores. Family Caregiving in an Aging Society: Policy Perspectives (Family Caregiver Applications series). California, USA: Sage Publications, Inc; 1995. p. 15-28.

53. Fernández-López JA, Fernández-Fidalgo M, Cieza A. Quality of life, health and well-being conceptualizations from the perspective of the International Classification of Functioning, disability and health (ICF). Rev Esp Salud Publica. 2010;84(2):169-84. DOI: 10.1590/s113557272010000200005

54. Addington-Hall J, Kalra L. Who should measure quality of life? BMJ. 2001;322(7299):1417-20. DOI: 10.1136/bmj.322.7299.1417

55. Sanjuás Benito C. Medición de la calidad de vida: ¿cuestionarios genéricos o específicos? Arch Bronconeumol. 2005;41(3):107-9. DOI: 10.1157/13071579

56. Sullivan AB, Miller D. Who is Taking Care of the Caregiver? J Patient Exp. 2015;2(1):7-12. DOI: $10.1177 / 237437431500200103$

57. Guardiola Wanden-Berghe R, Sanz-Valero J. Intervención psicológica en los cuidadores de los pacientes oncológicos menores de edad: revisión sistemática. Hosp Domic. 2021;5(1):45-4. DOI: 10.22585/hospdomic.v5i1.126

58. Piñol Jané A, Sanz Carrillo C. Importancia de la evaluación de la calidad de vida en atención primaria. Gastroenterol Hepatol. 2004;27(Supl 3):49-55.

59. Bernabeu-Martínez MA, Ramos Merino M, Santos Gago JM, Álvarez Sabucedo LM, WandenBerghe C, Sanz-Valero J. Guidelines for safe handling of hazardous drugs: A systematic review. PloS One. 2018;13(5):e0197172. DOI: 10.1371/journal.pone.0197172

60. Gea Cabrera A, Sanz-Lorente M, Sanz-Valero J, López-Pintor E. Compliance and Adherence to Enteral Nutrition Treatment in Adults: A Systematic Review. Nutrients. 2019;11(11):e2627. DOI: 10.3390/nu11112627 